\title{
OPEN Machine learning prediction of UV-Vis spectra features of organic compounds related to photoreactive potential
}

\begin{abstract}
Rafael Mamede, Florbela Pereira \& João Aires-de-Sousa ${ }^{\square}$
Machine learning (ML) algorithms were explored for the classification of the UV-Vis absorption spectrum of organic molecules based on molecular descriptors and fingerprints generated from 2D chemical structures. Training and test data ( $75 \mathrm{k}$ molecules and associated UV-Vis data) were assembled from a database with lists of experimental absorption maxima. They were labeled with positive class (related to photoreactive potential) if an absorption maximum is reported in the range between 290 and $700 \mathrm{~nm}$ (UV/Vis) with molar extinction coefficient (MEC) above $1000 \mathrm{Lmol}^{-1} \mathrm{~cm}^{-1}$, and as negative if no such a peak is in the list. Random forests were selected among several algorithms. The models were validated with two external test sets comprising 998 organic molecules, obtaining a global accuracy up to 0.89 , sensitivity of 0.90 and specificity of 0.88 . The ML output (UVVis spectrum class) was explored as a predictor of the 3T3 NRU phototoxicity in vitro assay for a set of 43 molecules. Comparable results were observed with the classification directly based on experimental UV-Vis data in the same format.
\end{abstract}

The UV-Vis absorption spectrum is a key physical property of an organic compound that determines many of its optoelectronic properties and photochemical reactivity. In the human body, the combined effect of an external chemical compound (e.g., plant toxins, phytomedicines, cosmetics, agrochemicals, food additives, dyes, drugs) and exposure to light, especially ultraviolet and visible radiation may give rise to an acute unwanted response in the skin or retina, which is called chemical phototoxicity ${ }^{1,2}$.

The prediction of UV-Vis spectra from the molecular structural formula is of general high interest to design new materials, identify potential phototoxic compounds, estimate missing spectroscopic data for known molecules, or curate databases of experimental spectra.

Machine learning (ML) techniques have been reported for the prediction of optical and photophysical properties of organic compounds $s^{3-6}$. Joung et al. ${ }^{3}$ reported a deep learning model developed with an experimental database of 30,094 chromophore/solvent combinations to predict several optical properties, namely, the first absorption peak position, bandwidth, and extinction coefficient, the emission peak position, bandwidth, and photoluminescence quantum yield; and illustrated the possibilities of applying ML to find target molecules with desired optical and photophysical properties. The root mean squared errors of the predicted values were found to be 26.6 and $28.0 \mathrm{~nm}$ for absorption and emission peak positions. A comparison between predictions of the absorption and emission spectra of coumarin 153 in ethanol using the ML model and TD-DFT calculations revealed a better performance of the ML model when compared to the theoretical calculations ${ }^{3}$. Another database of experimental and computational UV-Vis absorption spectra attributes was recently obtained through mining methods ${ }^{7}$.

ML algorithms can also be trained with theoretically calculated data sets obtained, e.g., by DFT methods, for faster estimation of molecular properties ${ }^{8-11}$. ML models based on theoretical optical spectra pre-calculated by finite-difference time-domain (FDTD) simulations for gold nanoparticles and nanorods were reported by Pashkov et al. ${ }^{4}$ The models were explored both to predict structural parameters for a given spectrum and to predict a spectrum for given structural parameters. Gosh et $a .^{5}$ calculated a database of $132 \mathrm{k}$ excitation spectra using the PBE density functional augmented with vdW corrections, and trained neural networks with various architectures to predict the spectra from the $3 \mathrm{D}$ structure. Kang et al. ${ }^{6}$ used random forests to predict the highest 
oscillator strength and associated excitation energy among ten excitation states of molecules from 1 and 2D descriptors of the molecule. The model was trained with the TD-DFT results of about half a million molecules.

Phototoxicity is strongly related to molecular photochemistry and photostability ${ }^{2}$. The optimization of ADME-Tox parameters (absorption, distribution, metabolism, excretion, and toxicology) using high-throughput tools is of great importance in drug discovery ${ }^{12}$, and ML approaches can be used to rationalize and predict phototoxicity, representing a valuable strategy for reducing experimental tests, if an acceptable level of accuracy of the developed models is ensured. Although several efforts have been reported to model phototoxicity directly from molecular structures ${ }^{13-17}$, the inclusion of spectroscopic information can improve predictive models ${ }^{2}$ and add chemically sound indicators that can be theoretically calculated or learned from more easily available and larger data sets.

Training ML models to predict full UV-Vis spectra requires large databases of spectra obtained under consistent conditions to predict multiple continuous variables (e.g., the molar extinction coefficients at several wavelengths). Differently, here we report the exploration of ML tools to classify organic molecules in terms of their UV-Vis absorption spectrum based on molecular descriptors.

The relationship between features of the UV-Vis absorption spectrum ("photoactivity") and phototoxicity can be clearly understood from the ICH S10 guidance on photosafety evaluation of pharmaceuticals ${ }^{18}$, according to which a molecule is potentially photoreactive if it absorbs light in the range between 290 and $700 \mathrm{~nm}$ (UV/ Vis) with molar extinction coefficient (MEC) greater than $1000 \mathrm{~L} \cdot \mathrm{mol}^{-1} \cdot \mathrm{cm}^{-1}$. Excitation of molecules by light can lead to generation of reactive oxygen species and this can be an indicator of phototoxicity potential ${ }^{18}$. If the substance does not have a MEC above $1000 \mathrm{~L} \cdot \mathrm{mol}^{-1} \cdot \mathrm{cm}^{-1}$ in the above-mentioned window no direct phototoxicity is anticipated in humans ${ }^{18}$.

We retrieved data from the Reaxys (https://www.reaxys.com) database ${ }^{19}$ for $>80,000$ molecules, and positive/ negative classes related to photoreactive potential were assigned from the lists of absorption maxima and molar extinction coefficients with threshold values based on the ICH S10 guidance.

An external data set of molecules for which data was available both for UV-Vis absorption ${ }^{19}$ and for in vitro phototoxicity assays ${ }^{2}$ was used to evaluate the overlap of correlations between a phototoxicity test and the UV-Vis spectrum class (experimental or predicted by ML). However, we would like to emphasize that this study aimed at training ML models to predict features of the UV-Vis spectra from the molecular structure, rather than predicting phototoxicity or evaluating the usefulness of spectroscopic data to predict phototoxicity.

\section{Methods}

Data sets/selection of training and test sets. Molecular structures were retrieved from the Reaxys database (https://www.reaxys.com) ${ }^{19}$ with associated UV-Vis absorption maxima and molar extinction coefficient (MEC) values and were filtered for molecular weight in the range 98-1080 g/mol, only one fragment, methanol as the solvent, exclusion of molecules with metal atoms, and restriction to publication date before 2016. The molecular structures were standardized by normalizing tautomerism, mesomerism and aromaticity using the Standardizer program version 19.19.0, ChemAxon (https://www.chemaxon.com). Duplicates were removed based on InChI identifiers and stereochemistry was not considered so that stereoisomers were considered as duplicates. Compounds with a non-zero global charge, radicals or valence errors were also discarded. The final data set comprises 74,784 molecules: 37,038 molecules assigned to the positive class (POS, molecules with one or more absorption maxima between $290-700 \mathrm{~nm}$ with $\mathrm{MEC} \geq 1000 \mathrm{Lmol}^{-1} \mathrm{~cm}^{-1}$ ) and the remaining 37,746 molecules assigned to the negative class (NEG). The definition of the classes was based on the ICH S10 guidance on photosafety evaluation of pharmaceuticals ${ }^{18}$.

The data set was randomly divided into a training set of 72,788 molecules (POS class: 36,036 molecules and NEG class: 36,752 molecules), a test set I of 998 molecules (POS class: 501 molecules and NEG class: 497 molecules), and a test set II of 998 molecules (POS class: 512 molecules and NEG class: 486 molecules). The test set II includes 43 molecules for which the result of the 3T3 NRU phototoxicity in vitro assay is also available from Schmidt et al. ${ }^{2}$ Table 1 shows the distribution of UV-Vis absorption features in the data sets.

Calculation of molecular descriptors and fingerprints. Molecular fingerprints and 1D\&2D molecular descriptors were calculated with PaDEL-Descriptor version $2.21^{20}$, and RDKit ${ }^{21}$. Different types of fingerprints with different sizes were calculated and explored: 166 MACCS (MACCS keys), 307 Substructure (presence and count of SMARTS patterns for Laggner functional group classification-Sub and SubC respectively), 881 PubChem fingerprints ${ }^{22}, 1024$ CDK (circular fingerprints), 1024 CDK extended (circular fingerprints with additional bits describing ring features), and 1024 MorganFP $^{23}$. The 1D\&2D molecular descriptors comprise 1443 descriptors, including electronic, topological, and constitutional descriptors.

Modified Distance Descriptors $(\mathrm{Md})^{10}$ are based on the molecular connectivity thus making no use of bond orders and atomic formal charges (avoiding the generation of a 3D conformer, the application of an aromaticity definition and the mesomerism standardization). The descriptors count the pairs of atoms in a molecule at specific "modified distances". Modified distances were defined in terms of the van der Waals radius of the atoms and Sanderson electronegativity of neighbors. Md descriptors were calculated for 1010 intervals, using a resolution of 0.017 , interatomic distances up to 4 bonds, and a distance factor of 4 .

Estimated molecular orbital energies $\left(E_{\mathrm{HOMO}}, E_{\mathrm{LUMO}}\right.$ and GAP) were obtained with previously in-house developed ML models trained with DFT calculated data ${ }^{10}-\mathrm{ML}$ quantum descriptors $\left(\mathrm{ML}_{\mathrm{QD}}\right)$. They include 10 values obtained for the three properties with different models.

The calculators for some types of descriptors/fingerprints did not process all molecules, and the corresponding training sets have slightly different sizes: 72,787 for RDKit and RDKit Morgan fingerprints; 72,771 for MACCS, Sub, SubC and PubChem fingerprints; 72,747 for 1D\&2D molecular descriptors; 72,770 for $\mathrm{ML}_{\mathrm{QD}}$ descriptors. 


\begin{tabular}{|c|c|c|c|}
\hline & Training set & Test set I & Test set II \\
\hline \multicolumn{4}{|l|}{ POS class $^{\mathrm{a}}$} \\
\hline $1000 \leq \mathrm{MEC} \leq 5000$ & 21.3 & 22.4 & 19.5 \\
\hline $5000 \leq \mathrm{MEC}<10,000$ & 24.0 & 23.3 & 25 \\
\hline MEC $\geq 10,000$ & 54.7 & 54.3 & 55.5 \\
\hline \multicolumn{4}{|l|}{ NEG class $^{\mathrm{b}}$} \\
\hline$\lambda<290 \mathrm{~nm}, \mathrm{MEC}<1000$ & 10.4 & 10.7 & 10.5 \\
\hline$\lambda<290 \mathrm{~nm}, \mathrm{MEC} \geq 1000$ & 91.1 & 88.9 & 91.6 \\
\hline$\lambda>700 \mathrm{~nm}, \mathrm{MEC}<1000$ & 0.005 & 0 & 0 \\
\hline$\lambda>700 \mathrm{~nm}, \mathrm{MEC} \geq 1000$ & 0.07 & 0.20 & 0 \\
\hline $290 \leq \lambda \leq 700 \mathrm{~nm}, \mathrm{MEC} \leq 900$ & 6.5 & 8.0 & 5.6 \\
\hline $290 \leq \lambda \leq 700 \mathrm{~nm}$, MEC $>900$ & 0.23 & 0.4 & 0.21 \\
\hline
\end{tabular}

Table 1. Distribution of UV-Vis absorption features in the data sets ( $\mathrm{MEC}$ values in $\left.\mathrm{Lmol}^{-1} \mathrm{~cm}^{-1}\right)$. ${ }^{\text {a }}$ Statistics concerning the peak with the highest MEC within the $290-700 \mathrm{~nm}$ window; ${ }^{\mathrm{b}}$ statistics concerning any listed peak.

Selection of descriptors. In the quest for QSPR models with reduced number of descriptors, descriptor selection was performed based on the importance of descriptors assessed by RF (mean decrease in accuracy measure) with the $\mathrm{R}$ program version 3.6.1. ${ }^{24}$.

Machine learning (ML) methods. Classification and Regression Trees (CART) ${ }^{25}$ operate by recursive partitioning of the initial data set with the goal of maximizing an information gain function (or variance reduction in regression trees) calculated for the various branches and terminal nodes. The best tree size is identified among sub-trees by cross-validation, or splits are not attempted if improvements above a threshold are not attained. Classification trees were built using the rpart package ${ }^{26}$ of the R program version 3.6.1 ${ }^{24}$ with default parameter values, except for $1 \mathrm{D} \& 2 \mathrm{D}$ descriptors (the cp parameter was set at 0.05 ).

Random forests $(\mathrm{RF})^{27}$ were implemented as ensembles of unpruned classification trees which are grown using bootstrap samples of the training set. Each individual tree is different because bootstrap samples vary, and randomly selected subsets of descriptors are made available for each node split. Predictions are obtained by a majority vote of the classification trees in the forest. An internal cross-validation error (or out-of-bag estimation, $\mathrm{OOB}$ ) is directly calculated with the prediction error for the objects left out in the bootstrap procedure. The importance of a descriptor can be assessed by the mean decrease in accuracy when the values of the descriptor are randomly permuted. A probability is assigned to every prediction based on the number of votes obtained by the predicted class. RFs were grown with the $\mathrm{R}$ program ${ }^{24}$, version 3.6.1, using the randomForest library ${ }^{28}$. The model was manually optimized recurring to the OOB estimation, with the number of trees from 500 to 1000 and the number of available descriptors for each rule (mtry) equal to the square root or $1 / 3$ of the total number of descriptors.

Support Vector Machines (SVM) ${ }^{29}$ map the training data into a hyperspace through a nonlinear mapping (a boundary or hyperplane) and then separate the classes of objects in this space. The boundary is positioned using examples in the training set-the support vectors. Kernel functions can be used to transform data into a hyperspace where the classes become linearly separable. In this study, SVM were implemented with the program Weka version $3.8 .3^{30}$, using the LIBSVM package ${ }^{31}$. The type of SVM was set to C-SVM-classification and the radial basis function was used for the kernel function. Hyper-parameter tuning was performed with the Experimenter application in Weka using ten-fold cross-validation. $\mathrm{C}$ and $\gamma$ values varied from 1 to 1000 and 0.003 to 0.0045 , respectively. The $\mathrm{C}$ and $\gamma$ values were finally set to 500 and 0.004 , respectively, and the other parameters were used with default values.

Deep Learning Multilayer Perceptron Networks $\left({ }_{d} M L P\right)$ were trained and applied with the software library Keras $^{32}$ version 2.2.5 based on Tensorflow numerical backend engine ${ }^{33}$. The feed-forward neural network architecture was manually optimized in terms of the number of hidden layers (2 to 6), weights initializer (random normal and Glorot uniform), optimizer (adadelta, adam, SGD, and SGD-nesterov), learning rate (0.0001 to 0.01 ) and decay (0 to 0.01$)$. The final hyper-parameter settings were selected for our study based on a best of 10 validation experiments with the training set-Table 2 .

Statistical measures of models' performance. Models were evaluated with external test sets and by internal validation with the training set. An OOB estimation for RF and a tenfold cross-validation for the other ML techniques procedures were employed with the training set. The following measures were calculated: true positives (TP), true negatives (TN), false positives (FP), false negatives (FN), sensitivity (SE), specificity (SP), overall predictive accuracy (Q) and Matthews correlation coefficient (MCC) were calculated with Eqs. (1)-(4). 


\begin{tabular}{|l|l|}
\hline Hyper-parameter & Setting \\
\hline Initializer & Random normal \\
\hline Number of hidden layers & 4 \\
\hline Number of neurons in the 1st and 2nd layers & 250 \\
\hline Number of neurons in the 3rd layer & 8 \\
\hline Number of neurons in the 4th layer & 4 \\
\hline Number of neurons in the 5th layer & 1 \\
\hline Activation 1st-4th layers & Relu \\
\hline Activation 5th layer & Sigmoid \\
\hline Batch size & 36 \\
\hline Optimizer & Adam \\
\hline Loss & Binary crossentropy \\
\hline Epochs & 100 \\
\hline Learning rate & 0.001 \\
\hline Decay & $10^{-6}$ \\
\hline
\end{tabular}

Table 2. Hyper-parameter settings of the best ${ }_{\mathrm{d}} \mathrm{MLP}$ model.

\begin{tabular}{|l|l|l|l|l|l|l|l|l|l|}
\hline Descriptors & $\mathbf{Q ~ T r}^{\mathbf{a}}$ & $\mathbf{Q}^{\mathbf{b}}$ & $\mathbf{S P}^{\mathbf{c}}$ & $\mathbf{S E}^{\mathbf{d}}$ & $\mathbf{M C C}^{\mathbf{e}}$ & $\mathbf{T P}^{\mathbf{f}}$ & $\mathbf{T N}^{\mathbf{g}}$ & $\mathbf{F P}^{\mathbf{h}}$ & $\mathbf{F N}^{\mathbf{i}}$ \\
\hline RDKitMorganFP & 0.88 & 0.88 & 0.88 & 0.88 & 0.76 & 441 & 435 & 62 & 60 \\
\hline ExtCDK & 0.89 & 0.87 & 0.86 & 0.9 & 0.75 & 448 & 425 & 72 & 53 \\
\hline CDK & 0.89 & 0.87 & 0.85 & 0.9 & 0.74 & 449 & 421 & 76 & 52 \\
\hline MACCS & 0.85 & 0.87 & 0.85 & 0.89 & 0.74 & 444 & 422 & 75 & 57 \\
\hline Md & 0.87 & 0.87 & 0.85 & 0.89 & 0.74 & 448 & 422 & 75 & 53 \\
\hline PubChem & 0.88 & 0.86 & 0.85 & 0.88 & 0.73 & 443 & 420 & 77 & 58 \\
\hline RDKitFP & 0.87 & 0.87 & 0.85 & 0.88 & 0.73 & 442 & 425 & 73 & 58 \\
\hline 1 D\&2D & 0.86 & 0.85 & 0.83 & 0.87 & 0.7 & 434 & 415 & 83 & 66 \\
\hline SubC & 0.85 & 0.84 & 0.81 & 0.86 & 0.67 & 430 & 404 & 93 & 71 \\
\hline Sub & 0.8 & 0.8 & 0.77 & 0.83 & 0.61 & 420 & 382 & 115 & 81 \\
\hline ML $_{\text {QD }}$ & 0.77 & 0.75 & 0.72 & 0.79 & 0.51 & 391 & 362 & 138 & 107 \\
\hline
\end{tabular}

Table 3. Evaluation of different molecular descriptors and fingerprints for the prediction of UV-Vis spectrum class using the RF algorithm. ${ }^{\mathrm{a}}$ Overall predictive accuracy for the training set in OOB estimation. ${ }^{\mathrm{b}}$ Overall predictive accuracy (test set I). ${ }^{~}$ Specificity (test set I). ${ }^{\mathrm{d}}$ Sensitivity (test set I). ${ }^{\mathrm{e}} \mathrm{MCC}$, Matthews correlation

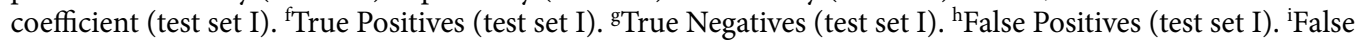
Negatives (test set I).

$$
\begin{gathered}
S E=\frac{T P}{T P+F N} \\
S P=\frac{T N}{T N+F P} \\
Q=\frac{T P+T N}{T P+F N+T N+F P} \\
M C C=\frac{T P X T N-F N X F P}{\sqrt{(T P+F N)(T P+F P)(T N+F N)(T N+F P)}}
\end{gathered}
$$

\section{Results and discussion}

Machine learning prediction of UV-Vis photoreactive potential. Several ways of representing the molecular structures were evaluated as input to RF classification models, which were trained to predict the UV-Vis spectrum class. The number of trees in the forest was set to 500, the number of descriptors available for each rule was the square root of the total number of descriptors and the other parameters were used with default values. The performance of the models was evaluated by internal validation with the training set (out-of-bag estimation, OOB) and by validation with test set I (998 molecules)-Table 3. 


\begin{tabular}{|c|c|c|c|c|c|c|c|c|c|}
\hline Descriptors & Q Tr ${ }^{a}$ & $Q^{b}$ & $\mathrm{SP}^{\mathrm{c}}$ & $\mathrm{SE}^{\mathrm{d}}$ & MCC $^{\mathrm{e}}$ & $T P^{f}$ & $\mathrm{TN}^{\mathrm{g}}$ & $\mathbf{F P}^{\mathrm{h}}$ & $\mathrm{FN}^{\mathrm{i}}$ \\
\hline $\mathrm{ExtCDK}+\mathrm{ML}_{\mathrm{QD}}$ & 0.89 & 0.88 & 0.86 & 0.90 & 0.76 & 452 & 428 & 69 & 49 \\
\hline ExtCDK+Md & 0.89 & 0.88 & 0.86 & 0.89 & 0.75 & 446 & 429 & 68 & 53 \\
\hline RDKitMorganFP + Md & 0.88 & 0.87 & 0.85 & 0.88 & 0.73 & 441 & 424 & 73 & 60 \\
\hline RDKitMorganFP $+\mathrm{ML}_{\mathrm{QD}}$ & 0.87 & 0.86 & 0.84 & 0.88 & 0.73 & 443 & 418 & 79 & 58 \\
\hline $1 \mathrm{D} \& 2 \mathrm{D}+\mathrm{ML}_{\mathrm{QD}}$ & 0.87 & 0.86 & 0.83 & 0.88 & 0.72 & 442 & 414 & 83 & 59 \\
\hline $\mathrm{Md}+\mathrm{ML}_{\mathrm{QD}}$ & 0.87 & 0.86 & 0.84 & 0.87 & 0.71 & 436 & 418 & 79 & 65 \\
\hline ExtCDK+ 1D\&2D & 0.87 & 0.86 & 0.84 & 0.87 & 0.71 & 438 & 416 & 81 & 63 \\
\hline $\mathrm{Md}+1 \mathrm{D} \& 2 \mathrm{D}$ & 0.87 & 0.86 & 0.85 & 0.86 & 0.71 & 432 & 422 & 75 & 69 \\
\hline RDKitMorganFP + 1D\&2D & 0.86 & 0.85 & 0.83 & 0.86 & 0.70 & 434 & 413 & 84 & 68 \\
\hline
\end{tabular}

Table 4. Evaluation of the performance of combined descriptors for the prediction of UV-Vis spectrum class using the RF algorithm. ${ }^{\mathrm{a} O v e r a l l ~ p r e d i c t i v e ~ a c c u r a c y ~ f o r ~ t h e ~ t r a i n i n g ~ s e t ~ i n ~ O O B ~ e s t i m a t i o n . ~}{ }^{\mathrm{b}}$ Overall predictive accuracy (test set I). ${ }^{c}$ Specificity (test set I). ${ }^{\mathrm{d} S e n s i t i v i t y ~(t e s t ~ s e t ~ I) . ~}{ }^{\mathrm{e}} \mathrm{MCC}$, Matthews correlation

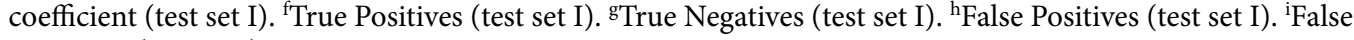
Negatives (test set I).

\begin{tabular}{|l|l|l|l|l|l|l|l|l|l|}
\hline Model & $\mathbf{Q ~ T r}^{\mathbf{a}}$ & $\mathbf{Q}^{\mathbf{b}}$ & $\mathbf{S P}^{\mathbf{c}}$ & $\mathbf{S E}^{\mathbf{d}}$ & $\mathbf{M C C}^{\mathbf{e}}$ & $\mathbf{T P}^{\mathbf{f}}$ & $\mathbf{T N}^{\mathbf{g}}$ & $\mathbf{F P}^{\mathbf{h}}$ & FN $^{\mathbf{i}}$ \\
\hline RF & 0.88 & 0.87 & 0.87 & 0.88 & 0.75 & 440 & 431 & 66 & 61 \\
\hline${ }_{\mathrm{d}} \mathrm{MLP}$ & 0.94 & 0.82 & 0.82 & 0.82 & 0.65 & 412 & 409 & 88 & 89 \\
\hline SVM & 0.87 & 0.84 & 0.82 & 0.85 & 0.68 & 428 & 410 & 87 & 75 \\
\hline
\end{tabular}

Table 5. Evaluation of alternative ML algorithms for the prediction of UV-Vis absorption spectrum class using 250 selected RDKitMorganFP molecular attributes. ${ }^{a}$ Overall predictive accuracy for the training set in OOB estimation. ${ }^{b}$ Overall predictive accuracy (test set I). ${ }^{c}$ Specificity (test set I). ${ }^{d}$ Sensitivity (test set I). ${ }^{\mathrm{e}}$ MCC, Matthews correlation coefficient (test set I). ${ }^{\text {f}}$ True Positives (test set I). ${ }^{\text {g}}$ True Negatives (test set I). ${ }^{\mathrm{h}}$ False

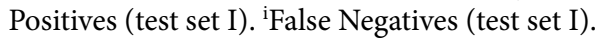

The models with CDK, ExtCDK, and RDKitMorganFP showed the best overall predictive accuracy for the training set in the OOB estimation and similar predictions were achieved for the test set. Both CDK and ExtCDK representations yielded slightly higher sensitivity than RDKitMorganFP, but the latter enabled the highest specificity and MCC of all models. It is also worth mentioning that the few $\mathrm{ML}_{\mathrm{QD}}$ descriptors alone provided results that, although worse, are still good.

The complementary potential of several molecular representations was investigated next by combining Md, ExtCDK, RDKitMorganFP, $1 \mathrm{D} \& 2 \mathrm{D}$ and $\mathrm{ML}_{\mathrm{QD}}$ molecular descriptors/fingerprints. The criteria for generating the combinations were the complementary nature of the attributes and, in case of similar sets, those yielding better predictions individually. The results in Table 4 show that combined descriptors did not provide any significantly superior model.

The impact of random fluctuations in the models was assessed by re-training with different seed initialization of random functions. Fluctuations of Q up to $2 \%$ were observed meaning that differences below this value cannot be considered significant. The best models were also validated with Y-scrambling experiments in which the percentage of correct predictions varied between $49.85 \%$ and $50.1 \%$ in 5 experiments.

The RDKitMorganFP model achieved the best results for the test set with a Q of 0.88 and a MCC of 0.76 (Table 3). The 250 most important fingerprint bits of the RDKitMorganFP were identified by the RF model for the training set and were selected for training a new RF model with lower computational cost, as well as other models with different ML algorithms (SVM and ${ }_{\mathrm{d}} \mathrm{MLP}$ ) - Table 5. Reduction of attributes yielded a RF model with essentially the same quality. The receiver operating characteristic curve (ROC) obtained for the test set I with the RF model trained with 250 RDKitMorganFP attributes is displayed in Fig. 1. Superior results could not be observed with the alternative ML algorithms.

The best model (RF 250 RDKitMorganFP) was further validated with the independent test set 2 . The statistical parameters of the obtained predictions are in line with those obtained for the first test set. Models trained with CDK and ExtCDK fingerprints (default number of features) were also evaluated (Table 6).

Analysis of important molecular attributes. Interpretable molecular descriptors (1D\&2D and $\mathrm{ML}_{\mathrm{QD}}$ ) and fingerprints (MACCS, PubChem and Sub) were processed with machine learning algorithms to provide information on relevant structural features for the UV-Vis spectrum classification. MACCS, PubChem and Sub fingerprints are binary attributes that encode the presence or absence of a particular structural feature. The importance of attributes calculated by RF was inspected. Additional to RF, simple classification trees were grown to understand relationships between individual attributes and the potential photoreactivity of molecules. As expected, the trees are poorer predictive models than the more complex RF (Table 7) but are useful to analyze 


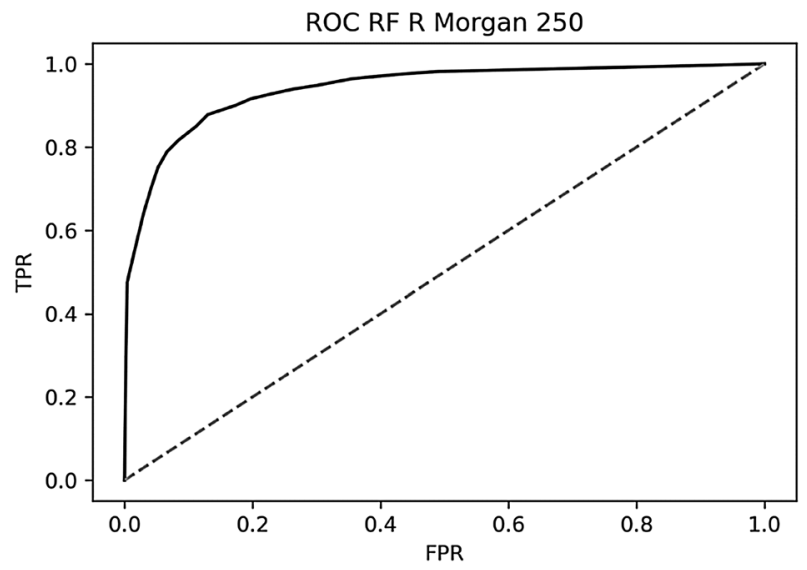

Figure 1. Receiver operating characteristic curve (ROC) obtained for the test set I with the RF model trained with 250 RDKitMorganFP attributes.

\begin{tabular}{|l|l|l|l|l|l|l|l|l|}
\hline Model & $\mathbf{Q}^{\mathbf{a}}$ & $\mathbf{S P}^{\mathbf{b}}$ & $\mathbf{S E}^{\mathbf{c}}$ & $\mathbf{M C C}^{\mathbf{d}}$ & $\mathbf{T P}^{\mathbf{e}}$ & $\mathbf{T N}^{\mathbf{f}}$ & $\mathbf{F P}^{\mathbf{g}}$ & $\mathbf{F N}^{\mathbf{h}}$ \\
\hline RDKitMorganFP & 0.89 & 0.88 & 0.90 & 0.78 & 454 & 432 & 60 & 52 \\
\hline ExtCDK & 0.89 & 0.87 & 0.91 & 0.78 & 460 & 428 & 64 & 46 \\
\hline CDK & 0.88 & 0.86 & 0.90 & 0.77 & 458 & 423 & 68 & 49 \\
\hline
\end{tabular}

Table 6. Evaluation of RF models trained with circular fingerprints to predict the UV-Vis absorption spectrum of organic molecules in test set II. ${ }^{\mathrm{a}}$ Overall predictive accuracy. ${ }^{\mathrm{b}}$ Specificity. ${ }^{\mathrm{c}}$ Sensitivity. ${ }^{\mathrm{d}} \mathrm{MCC}$, Matthews correlation coefficient. ${ }^{\mathrm{e}}$ True Positives. ${ }^{\mathrm{f}}$ True Negatives. ${ }^{\mathrm{g}} \mathrm{False}$ Positives. ${ }^{\mathrm{h}} \mathrm{False}$ Negatives.

\begin{tabular}{|l|l|l|l|l|l|l|l|l|}
\hline Model & $\mathbf{Q}^{\mathbf{a}}$ & $\mathbf{S P}^{\mathbf{b}}$ & $\mathbf{S E}^{\mathbf{c}}$ & $\mathbf{M C C}^{\mathbf{d}}$ & $\mathbf{T P}^{\mathbf{e}}$ & $\mathbf{T N}^{\mathbf{f}}$ & FP $^{\mathbf{g}}$ & FN $^{\mathbf{h}}$ \\
\hline 1D\&2D & 0.73 & 0.71 & 0.76 & 0.47 & 380 & 353 & 146 & 119 \\
\hline $\mathrm{ML}_{\mathrm{QD}}$ & 0.72 & 0.65 & 0.80 & 0.45 & 398 & 325 & 174 & 101 \\
\hline MACCS & 0.71 & 0.67 & 0.75 & 0.42 & 373 & 335 & 164 & 126 \\
\hline PubChem & 0.71 & 0.67 & 0.76 & 0.42 & 377 & 332 & 167 & 122 \\
\hline Sub & 0.69 & 0.68 & 0.70 & 0.38 & 349 & 339 & 160 & 150 \\
\hline
\end{tabular}

Table 7. Evaluation of classification trees based on interpretable fingerprints and molecular descriptors for the classification of UV-Vis absorption spectra of organic molecules in test set I. a Overall predictive accuracy for

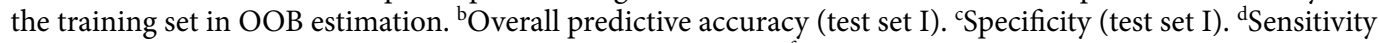

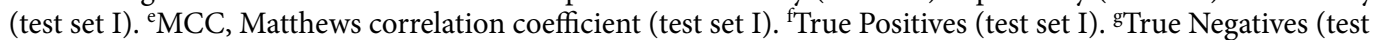

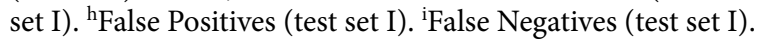

the importance of molecular fragments. The classification tree trained with PubChem fingerprints is shown in Fig. 2, and the trees obtained with the other attributes are in Figures S1-S4 of the Supplementary Material.

In the tree obtained with PubChem fingerprints (Fig. 2) the first two rules use the presence of two conjugated $\mathrm{C}=\mathrm{C}$ double bonds and the $\alpha, \beta$-unsaturated carbonyl in fragment " $\mathrm{O}=\mathrm{C}-\mathrm{C}=\mathrm{C}-\mathrm{C}$ " as discriminant features. Additionally, the presence of aromatic fragments with nitrogen or oxygen substituents ("N-C:C:C-C" and " $\mathrm{C}(\sim \mathrm{O})$ $(: C)(: C)$ ") were also used.

The features selected by the tree grown with MACCS fingerprints were similar and operated in similar ways. Features encoding the presence of aromaticity, double bonds connected to nitrogen atoms, as well as the absence of carbon atoms with at least two single bonds and at least two hydrogens were associated with the positive class.

Most of the inferred rules classify compounds as POS due to the presence of specific types of sub-structures, which agrees with the chemical knowledge that chromophores give rise to UV-Vis absorption. However, some rules associate the POS class with the absence of some aliphatic fragments. An example is the presence of a tertiary carbon atom; although it does not preclude the presence of chromophores in the molecule, a tertiary carbon atom is not involved in conjugation and is statistically associated with the negative class in our data set.

The 1D\&2D molecular descriptors enabled a tree (in Figure S3) to infer two powerful rules based on the number of atoms in the largest pi system. They discriminate molecules with extended conjugation and fused 


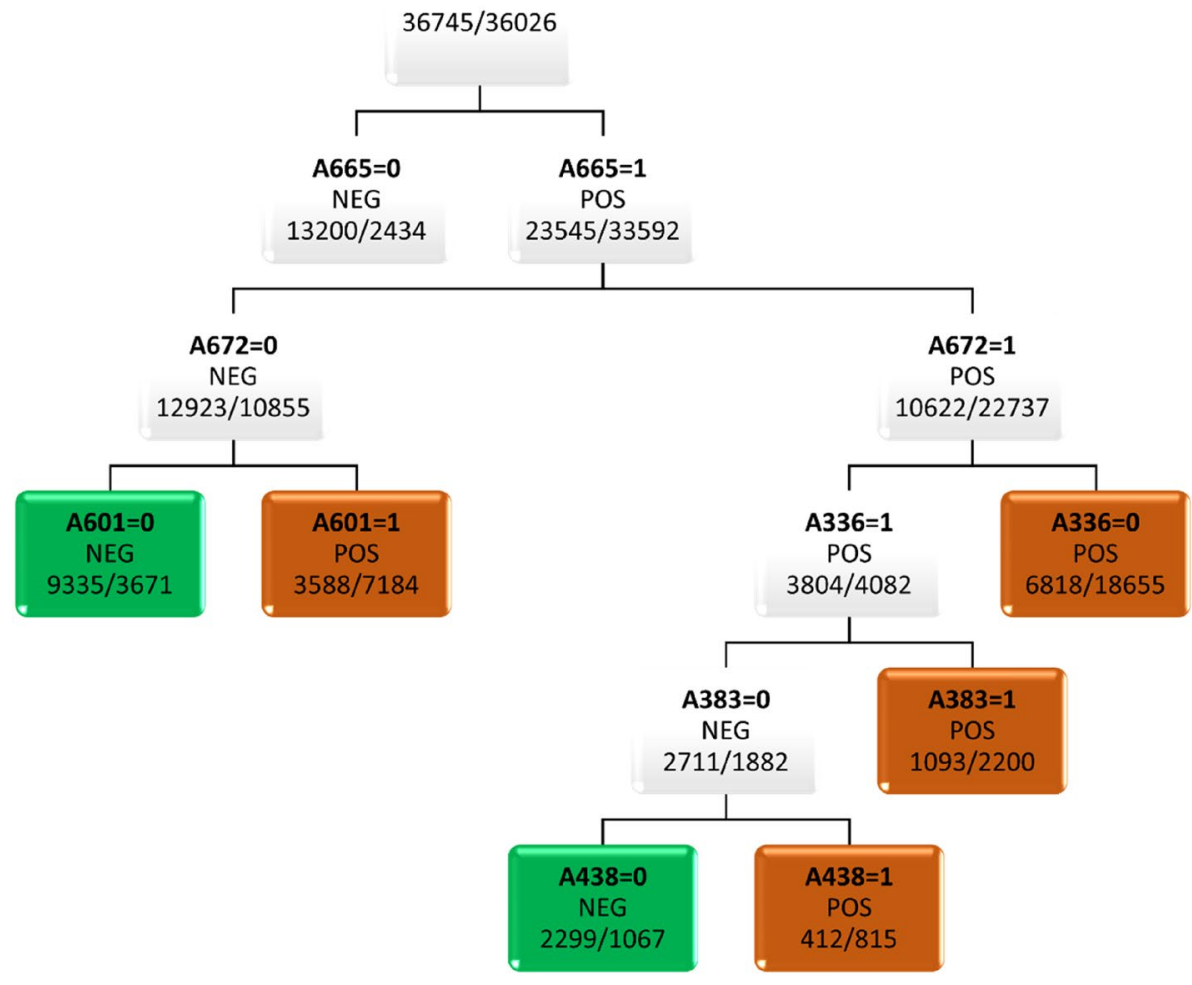

Figure 2. Classification tree based on PubChem fingerprints for the discrimination of molecules of the POS/ NEG classes related to potential photoreactivity. A665, C-C=C-C=C; A672, O=C-C=C-C; A601, N-C:C:C-C; A336, $\mathrm{C}(\sim \mathrm{C})(\sim \mathrm{C})(\sim \mathrm{C})(\sim \mathrm{H})$; A383, $\mathrm{C}(\sim \mathrm{O})(: \mathrm{C})(: \mathrm{C})$; A438, C $(-\mathrm{C})(-\mathrm{N})(=\mathrm{C})$.

bicyclic rings derived from e.g., naphthalene, indole, benzimidazole, benzoxazole, benzofuran, benzothiophene, or benzazepine. An additional rule is based on features of nitrogen atoms with two aromatic bonds.

Based exclusively on the estimated energies of the HOMO and LUMO orbitals, and their gap, a single rule was established that associates the POS class to GAP $<4.626 \mathrm{eV}$. Inspection of the database revealed that the molecules with the lowest value for this descriptor $(\sim 2 \mathrm{eV})$ include highly conjugated aromatic systems, such as tetracarboxdiimide derivatives, also corresponding to visible light absorption in the range between 500 and $700 \mathrm{~nm}$ with high MEC values $\left(>1000 \mathrm{Lmol}^{-1} \mathrm{~cm}^{-1}\right.$ ).

The ten most important attributes according to the MeanDecreaseGini parameter in the RF models are reported in Tables S1-S4 of the Supplementary Material for various fingerprints and descriptors. The most relevant attributes identified by the RF models are in line with those selected to build the trees, namely attributes accounting for the presence of aromaticity, unsaturation and conjugated systems. The importance of conjugation was highlighted by the selection of three Sub fingerprints that encode the presence of $\alpha, \beta$-unsaturated carbonyl or carboxyl groups. The presence of tertiary carbon atoms is at the top ten in three models (MACCS, Sub and PubChem-Tables S1-S3). This feature was also used in the tree of Fig. 2 associated with the negative class. Although some of the 1D\&2D descriptors have no straightforward interpretation, it is clear that different aspects of unsaturated systems are encoded by several of the most important attributes: number of atoms in the largest pi system, ratio of total conventional bond order with total path count, fraction of $\mathrm{sp}^{3}$ carbons to $\mathrm{sp}^{2}$ carbons, measure of relative unsaturation content, total number of bonds that have bond order greater than one. Furthermore, measures of global electronic features appear as highly relevant in positions 8 and 9.

Analysis of outliers. The RF model trained with all RDKitMorgan fingerprints predicted the test set I with accuracy of 0.88 and MCC 0.76 . The ROC curve of Fig. 1 illustrates the significance of the probabilities assigned by the RF models to the predictions. Among the 998 predicted molecules, the $15 \mathrm{FP}$ and $18 \mathrm{FN}$ with a probability higher than 0.8 were manually inspected to discover possible reasons for wrong predictions with high assigned probabilities. Most false negatives ( 12 out of $18 \mathrm{FN}$ ) correspond to molecules with peak wavelengths inside the photoreactivity window, but close to the lower endpoint of the interval (290-317 nm). Other 2 FN have a peak within the window but with a MEC value lower than $1500 \mathrm{Lmol}^{-1} \mathrm{~cm}^{-1}$. The other $4 \mathrm{FN}$ are compounds with peaks inside the window and high values of MEC reported in the database: a 16-membered macrolide with 3 deoxy sugar moieties attached and including a pi system $(\mathbf{1})^{34}$, two cyclic compounds with conjugated systems $\left(2^{35}\right.$ and $\left.3^{36}\right)$ and a quinazolinone connected to a thiazole $(4)^{37}$, Fig. 3.

Three of these four predictions could be explained by similar molecules in the training set, which were assigned to the NEG class based on the experimental data. The structures 1, 2, 3 and 4 in Fig. 3 were subjected to a similarity search against the training set, using fingerprints and Tanimoto coefficients. Molecules 1, 2 and 


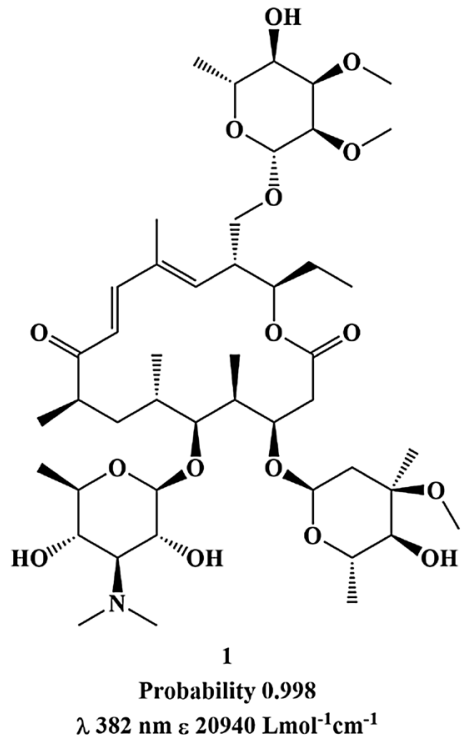

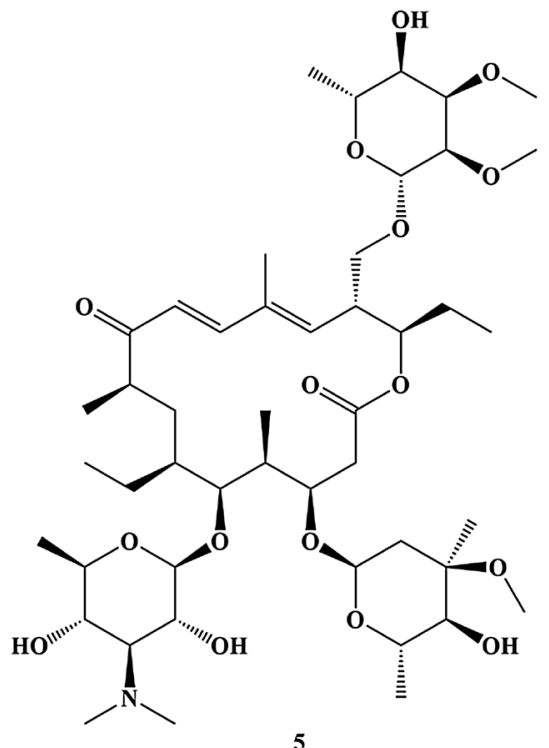

Class NEG OOB-Pred NEG $\lambda 282 \mathrm{~nm} \& 19400 \mathrm{Lmol}^{-1} \mathrm{~cm}^{-1}$<smiles>CCCCC(C)(C)c1ccc(O)c(O)c1</smiles>

$\lambda 525 \mathrm{~nm} \& 1345 \mathrm{Lmol}^{-1} \mathrm{~cm}^{-1}$<smiles>CC(=O)CC1=C2CCCC2=CC(OC(C)=O)(OC(C)=O)C1=O</smiles>

Probability 0.840

$\lambda 328 \mathrm{~nm} \& 3887 \mathrm{Lmol}^{-1} \mathrm{~cm}^{-1}$<smiles>Cc1cnc(-n2c(C)nc3c(Cl)cc(Cl)cc3c2=O)s1</smiles>

Probability 0.852

$\lambda 324 \mathrm{~nm} \& 40738 \mathrm{Lmol}^{-1} \mathrm{~cm}^{-1}$<smiles>O=CCc1ccc(O)c(O)c1</smiles>

Class NEG OOB-Pred NEG $\lambda 262 \mathrm{~nm} \& 8181 \mathrm{Lmol}^{-1} \mathrm{~cm}^{-1}$<smiles>CC(=O)OC1(OC(C)=O)C=C(C(C)(C)C)C=C(CCO)C1=O</smiles>

7

Class POS OOB-Pred POS $\lambda 322.6 \mathrm{~nm} \& 3234 \mathrm{Lmol}^{-1} \mathrm{~cm}^{-1}$<smiles>Cc1cnc(-n2c(C)nc3ccc(Cl)cc3c2=O)s1</smiles>

Class NEG OOB-Pred NEG $\lambda 240 \mathrm{~nm} \& 12302 \mathrm{Lmol}^{-1} \mathrm{~cm}^{-1}$

Figure 3. The chemical structures of four FN (1-4) predicted with high probability and their most similar training set counterpart structures (5-8). Experimental data is included as retrieved from the database.

4 have a similar counterpart in the training set assigned to the NEG class and predicted as NEG in the OOB estimation: molecules $5^{34}$ (Tanimoto coefficient of 0.99 ), $6^{38}$ (Tanimoto coefficient of 0.70 ) and $8^{37}$ (Tanimoto coefficient of 0.94 ), respectively. Compound 3 has a similarity of 0.83 with compound $7^{36}$ of the training set, and both were assigned to the POS class based on the experimental data.

Although compound $\mathbf{5}$ is correctly assigned to the NEG class based on the experimental data, according to our definition of the POS and NEG classes (it has no maxima in the relevant window with MEC above the threshold), it represents in fact a borderline situation because UV-Vis absorption peaks are typically broad. Compound 5 has a peak at $282 \mathrm{~nm}$ with a MEC value of $19,400 \mathrm{Lmol}^{-1} \mathrm{~cm}^{-1}$; it is therefore highly probable that the MEC value at $290 \mathrm{~nm}$ is higher than $1000 \mathrm{Lmol}^{-1} \mathrm{~cm}^{-1}$ suggesting photoreactive potential. This example highlights a limitation of the models due to the nature of the experimental data here used (consisting in lists of UV-Vis absorption maxima): the absence (in the list) of a maximum within the relevant window does not guarantee that there is no absorptivity in the window with a MEC above the threshold. It may happen that the experimental spectrum did not cover the full window or the source publication reported only the highest peak(s), and there are often absorptivities in the window with a MEC above the threshold from bands whose maxima are outside the window. The similarity of compound $\mathbf{6}$ (NEG) to compound $\mathbf{2}$ may explain the prediction of the latter as negative; but inspection of the original source for compound 2 reveals that the reported UV-Vis data are for a 


\begin{tabular}{|l|l|l|l|l|}
\hline & \multicolumn{2}{|l|}{$\begin{array}{l}\text { Predicted } \\
\text { UV-Vis } \\
\text { spectrum } \\
\text { class }\end{array}$} & \multicolumn{2}{l|}{$\begin{array}{l}\text { Experimental } \\
\text { UV-Vis } \\
\text { spectrum } \\
\text { class }\end{array}$} \\
\cline { 2 - 5 } & POS & NEG & POS & NEG \\
\hline Toxic & 16 & 3 & 15 & 4 \\
\hline Non-toxic & 15 & 9 & 12 & 12 \\
\hline
\end{tabular}

Table 8. Confusion matrices relating RF-predicted and experimental UV-Vis spectrum class with the 3T3 NRU phototoxicity in vitro assay.

metal complex and shall not be considered for structure 2. This case illustrates how the method can be useful for the curation of experimental databases. Compound $\mathbf{8}$ may explain the false negative prediction for compound 4; the inclusion of an additional chlorine substituent in the aromatic ring added a new absorption band at a higher wavelength $^{37}$, and the ML model apparently did not learn that effect.

Concerning the $15 \mathrm{FP}$, it was observed that 4 molecules are among those in the NEG class with a peak at a wavelength only slightly below $290 \mathrm{~nm}(281-289.5 \mathrm{~nm})$ and with a high MEC value. Other 3 FP have peaks at wavelengths between 269 and $277 \mathrm{~nm}$ with MEC values between 9000 and $28,183 \mathrm{Lmol}^{-1} \mathrm{~cm}^{-1}$. Two FP have a peak with both the wavelength and MEC very near the thresholds (wavelength 295-306 nm, MEC 661-891 $\mathrm{Lmol}^{-1} \mathrm{~cm}^{-1}$ ). For the other $4 \mathrm{FP}$, all the MEC values listed in the database are between 3 and 5, which suggests they were originally reported in a different unit or as $\log (\mathrm{MEC})$ - confirmation was possible for at least one of them that a $\log (\mathrm{MEC})$ value was retrieved from the original literature as MEC and the compound exhibits indeed significant absorption within the $290-700 \mathrm{~nm}$ window. Finally, the other $2 \mathrm{FP}$ arise from a situation similar to compounds 4 and 8: a similarity search against the training set revealed that the inclusion/changing of a substituent in the aromatic system (in these cases to include methoxy and amino groups) is associated with the reporting of an absorption maxima at higher wavelengths.

UV-Vis spectrum classification as a predictor of in vitro phototoxicity. For 43 molecules of test set II, additional data is available concerning the 3 T3 NRU in vitro phototoxicity assay (PIV) ${ }^{2}$. The UV-Vis spectrum class was predicted for this subset with global accuracy 0.86 , sensitivity 0.96 and specificity 0.69 (comparing to $0.89,0.90$ and 0.88 , respectively, for the whole test set II-Table 6).

The RF output (UV-Vis spectrum class) was evaluated as a predictor of phototoxicity. Similarly, predictions of phototoxicity were also obtained using the lists of peaks and their MEC values available in the database of experimental data ("experimental spectrum class"). This enables to compare two approaches to the assessment of phototoxicity: (a) classification of the UV-Vis spectrum from lists of peaks available in the chemical literature, and (b) machine learning prediction of the spectrum class from the molecular structural formula. The confusion matrices are in Table 8.

The two confusion matrices are quite similar. The RF classification would correctly estimate 25 out of 43 molecules comparing to 27 using the experimental data. All the 12 non-toxic molecules assigned to the positive class in the experimental database were also predicted as positive by the RF model, and two toxic molecules were classified as negative both in the database and in the RF predictions. This suggests that a RF classification model for UV-Vis absorption features can assist in the estimation of in vitro toxicity similarly to experimental UV-Vis data. However, it must be emphasized that this study was not based on full spectra, but on lists of peaks extracted from the literature with their inherent incompleteness, and they certainly include noise.

The large number of non-toxic molecules assigned to the positive class is a limitation of UV-Vis spectra as a predictor of phototoxicity, because other characteristics of a chemical compound, beyond light absorption, are critical for phototoxicity, namely the ability to generate a reactive species. In any case, from the perspective of photosafety evaluation, high sensitivity is more important than specificity since molecules predicted as positive would be subjected to further experimental tests.

\section{Conclusion}

The random forest algorithm, trained with 72,787 organic molecules represented by Morgan circular fingerprints, was able to classify molecules according to UV-Vis spectrum features related to photoreactive potential with accuracy up to 0.89 , sensitivity of 0.90 and specificity of 0.88 for an independent test set of 998 molecules. The classes in the training and test sets were assigned based on data retrieved from the chemical literature, which consists of lists of absorption maxima with molar extinction coefficients.

Application of machine learning algorithms with interpretable molecular descriptors and fingerprints provided information on relevant structural features for the classification. The rules inferred by classification trees and the importance of attributes calculated by RF revealed that aromaticity, unsaturation, conjugation, and heteroatom substituents play an important role in discriminating between positive and negative classes.

Analysis of outliers (wrongly predicted molecules with high associated RF probability) highlighted three main situations: (a) absorption maxima with wavelengths near the lower endpoint of the established interval (290-700 nm) and/or MEC values close to the established threshold; (b) data noise, e.g., retrieval of log(MEC) value instead of MEC value; (c) insufficient learning of the impact of some heteroatom substituents on the absorption maxima. 
The ML assignment of molecules to the positive class (related to photoreactive potential) was a predictor of a positive outcome of the 3T3 NRU phototoxicity in vitro assay with a sensitivity of 0.84 and specificity of 0.38 in a test set of 43 molecules. Comparable results were observed with the assignment based on the experimental data available for the same set (sensitivity 0.79 and specificity 0.5 ). The results illustrate the potential of machine learning algorithms for the classification of molecules according to the UV-Vis absorption spectrum, to assist in photosafety evaluation.

\section{Data availability}

The dataset used in this study is provided by Elsevier Limited via the Reaxys database under license. The list of molecules is provided in the Supplementary Material in the SMILES notation with the corresponding Reaxys registry numbers; the Reaxys data is copyright (C) 2020 Elsevier Limited except certain content provided by third parties.

Received: 28 July 2021; Accepted: 22 November 2021

Published online: 09 December 2021

\section{References}

1. Onoue, S. et al. Chemical photoallergy: Photobiochemical mechanisms, classification, and risk assessments. J. Dermatol. Sci. 85, 4-11. https://doi.org/10.1016/j.jdermsci.2016.08.005 (2017).

2. Schmidt, F. et al. Computational investigation of drug phototoxicity: Photosafety assessment, photo-toxophore identification, and machine learning. Chem. Res. Toxicol. 32, 2338-2352. https://doi.org/10.1021/acs.chemrestox.9b00338 (2019).

3. Joung, J. F. et al. Deep learning optical spectroscopy based on experimental aatabase: Potential applications to molecular design. JACS Au. 1, 427-438. https://doi.org/10.1021/jacsau.1c00035 (2021).

4. Pashkov, D. M. et al. Quantitative analysis of the UV-Vis spectra for gold nanoparticles powered by supervised machine learning. J. Phys. Chem. C 125, 8656-8666. https://doi.org/10.1021/acs.jpcc.0c10680 (2021).

5. Ghosh, K. et al. Deep learning spectroscopy: Neural networks for molecular excitation spectra. Adv. Sci. 6, 1801367. https://doi. org/10.1002/advs.201801367 (2019).

6. Kang, B., Seok, C. \& Lee, J. Prediction of molecular electronic transitions using random forests. J. Chem. Inf. Model. 60(12), 5984-5994. https://doi.org/10.1021/acs.jcim.0c00698 (2020).

7. Beard, E. J., Sivaraman, G., Vazquez-Mayagoitia, A., Vishwanath, V. \& Cole, J. M. Comparative dataset of experimental and computational attributes of UV/vis absorption spectra. Sci. Data 6, 307. https://doi.org/10.1038/s41597-019-0306-0 (2019).

8. Zhang, Q. et al. A QSPR approach for the fast estimation of DFT/NBO partial atomic charges. Chemom. Intell. Lab. Syst. 134, 158-163. https://doi.org/10.1016/j.chemolab.2014.03.011 (2014).

9. Qu, X., Latino, D. A. R. S. \& Aires-de-Sousa, J. A big data approach to the ultra-fast prediction of DFT-calculated bond energies. J. Cheminformatics 5, 34. https://doi.org/10.1186/1758-2946-5-34 (2013).

10. Pereira, F. et al. Machine learning methods to predict density functional theory B3LYP energies of HOMO and LUMO orbitals. J. Chem. Inf. Model. 57, 11-21. https://doi.org/10.1021/acs.jcim.6b00340 (2017).

11. Pereira, F. \& Aires-de-Sousa, J. Machine learning for the prediction of molecular dipole moments obtained by density functional theory. J. Cheminformatics 10, 43. https://doi.org/10.1186/s13321-018-0296-5 (2018).

12. Bhhatarai, B., Walters, W. P., Hop, C., Lanza, G. \& Ekins, S. Opportunities and challenges using artificial intelligence in ADME/ Tox. Nat. Mater. 18, 418-422. https://doi.org/10.1038/s41563-019-0332-5 (2019).

13. Veith, G. D., Mekenyan, O. G., Ankley, G. T. \& Call, D. J. A QSAR analysis of substituent effects on the photoinduced acute toxicity of PAHs. Chemosphere 30, 2129-2142. https://doi.org/10.1016/0045-6535(95)00076-k (1995).

14. Ribeiro, F. A. D. \& Ferreira, M. M. C. QSAR model of the phototoxicity of polycyclic aromatic hydrocarbons. Comput. Theor. Chem. 719, 191-200. https://doi.org/10.1016/j.theochem.2005.01.026 (2005).

15. Peukert, S. et al. A method for estimating the risk of drug-induced phototoxicity and its application to smoothened inhibitors. MedChemComm 2, 973-976. https://doi.org/10.1039/c1md00144b (2011).

16. Ringeissen, S. et al. Development of a mechanistic SAR model for the detection of phototoxic chemicals and use in an integrated testing strategy. Toxicol. In Vitro 25, 324-334. https://doi.org/10.1016/j.tiv.2010.09.017 (2011).

17. Haranosono, Y., Kurata, M. \& Sakaki, H. Establishment of an in silico phototoxicity prediction method by combining descriptors related to photo-absorption and photo-reaction. J. Toxicol. Sci. 39, 655-664. https://doi.org/10.2131/jts.39.655 (2014).

18. ICH. S10 Photosafety evaluation of chemicals. https://database.ich.org/sites/default/files/S10_Guideline.pdf (2012).

19. Copyright (C) 2020 Elsevier Limited except certain content provided by third parties. Reaxys is a trademark of Elsevier Limited.

20. Yap, C. W. PaDEL-Descriptor: An open source software to calculate molecular descriptors and fingerprints. J. Comput. Chem. 32, 1466-1474. https://doi.org/10.1002/jcc.21707 (2011).

21. Landrum, G. RDKit: Open-Source Cheminformatics Software. http://www.rdkit.org (2016).

22. PubChem fingerprints. ftp://ftp.ncbi.nlm.nih.gov/pubchem/specifications/pubchem_fingerprints.txt (2009).

23. Rogers, D. \& Hahn, M. Extended-Connectivity Fingerprints. J. Chem. Inf. Model. 50, 742-754. https://doi.org/10.1021/ci100050t (2010).

24. R: A Language and Environment for Statistical Computing. R Foundation for Statistical Computing, Vienna, Austria, http:// www.R-project.org (2014).

25. Breiman, L., Friedman, J. H., Olshen, R. A. \& Stone, C. J. Classification and Regression Trees (Chapman \& Hall/CRC, 1984).

26. Therneau, T., Atkinson, B. \& Ripley B. rpart package: Recursive partitioning for classification, regression and survival trees. v. 4.1-15, https://cran.r-project.org/web/packages/rpart (1999-2019).

27. Breiman, L. Random forests. Mach. Learn. 45, 5-32. https://doi.org/10.1023/a:1010933404324 (2001).

28. Liaw, A. \& Wiener, M. Classification and Regression by randomForest. R News 2, 18-22 (2002).

29. Cortes, C. \& Vapnik, V. Support-Vector networks. Mach. Learn. 20, 273-297. https://doi.org/10.1023/a:1022627411411 (1995).

30. Frank, E., Hall, M. A. \& Witten, I. H. (2016). The WEKA Workbench. Online Appendix for Data Mining: Practical Machine Learning Tools and Techniques, Morgan Kaufmann, Fourth Edition, https://www.cs.waikato.ac.nz/ml/weka/Witten_et_al_2016_appen dix.pdf (2016).

31. Chang, C.-C. \& Lin, C.-J. LIBSVM: A library for support vector machines. ACM Trans. Intell. Syst. Technol. 2, 27. https://doi.org/ $10.1145 / 1961189.1961199(2011)$.

32. Chollet, F. et al. Keras, https://keras.io (2015).

33. Abadi, M. et al. TensorFlow: Large-scale machine learning on heterogeneous distributed systems. http://arxiv.org/abs/1603.04467 (2016).

34. Mallams, A. K. \& Rossman, R. R. Novel 3-0-a-L-cladinosyl sixteen-membered macrolide antibacterials. J. Chem. Soc. Perkin Trans. I(24), 799-805. https://doi.org/10.1039/P19890000799 (1989). 
35. Pascaly, M. et al. The systematic influence of tripodal ligands on the catechol cleaving activity of iron(III) containing model compounds for catechol 1,2-dioxygenases. J. Chem. Soc. Dalton Trans. 2001, 828-837. https://doi.org/10.1039/b0085111 (2001).

36. Quinkert, G. et al. Stereoselective ring opening of electronically excited cyclohexa-2,4-dienones: Cause and effect. Helv. Chim. Acta 80, 1683-1772. https://doi.org/10.1002/hlca.19970800602 (1997).

37. Párkányi, C. \& Schmidt, D. S. Synthesis of 5-chloro-2-methyl-3-(5-methylthiazol-2-yl)-4(3H)-quinazolinone and related compounds with potential biological activity. J. Heterocycl. Chem. 37, 725-729. https://doi.org/10.1002/jhet.5570370409 (2000).

38. Li, S. W., Spaziano, V. T. \& Burke, W. J. Synthesis of a biochemically important aldehyde, 3,4-dihydroxyphenylacetaldehyde. Bioorg. Chem. 26(1), 45-50. https://doi.org/10.1006/bioo.1998.1087 (1998).

\section{Acknowledgements}

This work was supported with financial support from Fundação para a Ciência e Tecnologia (FCT, MCTES) Portugal, under Grant UIDB/50006/2020 and UIDP/50006/2020 (provided to the Associate Laboratory for Green Chemistry LAQV). F.P. acknowledges Fundacão para a Ciência e a Tecnologia (FCT, MCTES) for the Norma transitória DL 57/2016 Program Contract. The work was also supported by the Radiation Biology and Biophysics Doctoral Training Programme (RaBBiT, PD/00193/2012); Applied Molecular Biosciences UnitUCIBIO (UIDB/04378/2020) and CEFITEC Unit (UIDB/00068/2020); and scholarship Grant Number PD/ BD/135475/2017 to R.M. JAS thanks David Ponting and co-workers at Lhasa Limited for useful suggestions and discussions.

\section{Author contributions}

R.M. and F.P. gathered the data, calculated the descriptors and trained the models. J.A.S. planned and coordinated the work. All authors reviewed the manuscript.

\section{Competing interests}

The authors declare no competing interests.

\section{Additional information}

Supplementary Information The online version contains supplementary material available at https://doi.org/ 10.1038/s41598-021-03070-9.

Correspondence and requests for materials should be addressed to J.A.-d.

Reprints and permissions information is available at www.nature.com/reprints.

Publisher's note Springer Nature remains neutral with regard to jurisdictional claims in published maps and institutional affiliations.

(f) Open Access This article is licensed under a Creative Commons Attribution 4.0 International License, which permits use, sharing, adaptation, distribution and reproduction in any medium or format, as long as you give appropriate credit to the original author(s) and the source, provide a link to the Creative Commons licence, and indicate if changes were made. The images or other third party material in this article are included in the article's Creative Commons licence, unless indicated otherwise in a credit line to the material. If material is not included in the article's Creative Commons licence and your intended use is not permitted by statutory regulation or exceeds the permitted use, you will need to obtain permission directly from the copyright holder. To view a copy of this licence, visit http://creativecommons.org/licenses/by/4.0/.

(c) The Author(s) 2021 